\title{
Lire la correspondance de Stendhal, textes édités par Martine Reid et Elaine Williamson
}

\section{Michel Arrous}

\section{(2) OpenEdition}

1 Journals

\section{Édition électronique}

URL : http://journals.openedition.org/studifrancesi/6678

DOI : 10.4000/studifrancesi.6678

ISSN : 2421-5856

Éditeur

Rosenberg \& Sellier

\section{Édition imprimée}

Date de publication : 1 septembre 2010

Pagination : 381-383

ISSN : 0039-2944

\section{Référence électronique}

Michel Arrous, «Lire la correspondance de Stendhal, textes édités par Martine Reid et Elaine

Williamson », Studi Francesi [En ligne], 161 (LIV | II) | 2010, mis en ligne le 30 novembre 2015, consulté

le 08 janvier 2021. URL : http://journals.openedition.org/studifrancesi/6678 ; DOI : https://doi.org/

10.4000/studifrancesi.6678

Ce document a été généré automatiquement le 8 janvier 2021.

\section{(c)}

Studi Francesi è distribuita con Licenza Creative Commons Attribuzione - Non commerciale - Non opere derivate 4.0 Internazionale. 


\title{
Lire la correspondance de Stendhal, textes édités par Martine Reid et Elaine Williamson
}

\author{
Michel Arrous
}

\section{RÉFÉRENCE}

AA.VV., Lire la correspondance de Stendhal, textes édités par Martine REID et Elaine WILLIAMSON, Paris, Champion, 2007, pp. 261.

1 Martine ReID et Elaine W ILLIAMson rappellent dans leur avant-propos que la correspondance de Stendhal, bien que moins connue que celle de Flaubert, offre cependant «une mosaïque d'états, de sentiments, de curiosités et de compétences multiples». Classées chronologiquement, les communications au colloque qui s'est tenu en décembre 2006 à l'University of London Institute in Paris ont saisi sur le vif un Stendhal qui pense, travaille, écrit et vit.

Préoccupée par la question des femmes chez Stendhal, Lucie GARNIER ( On ne naît pas femme, on le devient»: les lettres à Pauline et la condition féminine, pp. 11-25) relit les «tartines» à la cara sorella à la lumière du Deuxième Sexe. Qu'il s'agisse d'éducation, de littérature ou de société, le jeune Beyle s'efforce d' «appréhender le monde au féminin» quand, à propos du mariage, il explique - non sans paradoxes à l'avantage des hommes - que les conditions socio-culturelles sont autrement déterminantes que les conditions biologiques. Cette posture qui n'évoluera pas expliquerait la création de personnages féminins s'affranchissant des contraintes sociales, les «héroïnes rebelles» comme les a nommées R. Bolster. Ayant déjà évoqué ces mêmes lettres dans leur rapport à l'autobiographie, Béatrice DIDIER (Journal intime et correspondance: les lettres à Pauline, pp. 27-41) les considère cette fois dans la perspective du «Journal littéraire». Convergences et différences apparaissent entre les deux modes d'écriture du Moi pendant les années 1800-1805, au cours desquelles la correspondante privilégiée reçut 
des «lettres de direction», formulant un programme intellectuel, et des «lettres de confidence», apparentées au journal intime. Les lettres sont une «doublure du journal», à ceci près que dans certaines Beyle donne de sa vie de rassurantes et lénifiantes versions, d'où de troublants rapprochements: «Le moi ne peut-être un». La "passion» de Stendhal pour la littérature suppose une véritable «métaphysique littéraire», sans transcendance aucune, que Catherine MARIETTE retrouve dans ces mêmes lettres interprétées comme «une scène de lecture, passive et active à la fois» (Qu'est-ce que la «métaphysique littéraire»?, pp. 43-56). Se préparant rationnellement à devenir un «grand homme», l'apprenti écrivain lit, copie, fait des listes et des extraits. Le génie se conquiert. La «métaphysique littéraire» permet de penser la littérature et de travailler sur soi.

3 Avec l'exemple «modeste, circonscrit mais significatif» de la correspondance avec Faure et Bigillion entre mai 1805 et février 1806, à propos du drame de Victorine Bigillion que sa famille dut interner, Marie-Rose CORREDOR (Aux sources du discours clinique: la correspondance avec Félix Faure et François Bigillion, pp. 57-63) donne une remarquable analyse du discours clinique de Stendhal qui, à partir des symptômes décrits par F. Faure comprend que Victorine est à la fois victime de son furor et du regard social, alors qu'il ne se mettra à lire Pinel qu'en janvier 1806. Ces conjectures, acceptées par F. Faure dont la sœur fut elle aussi momentanément victime de troubles mentaux, montrent que Stendhal, dans le cas précis de la folie ordinaire de Victorine, ne s'est pas contenté de réfléchir «d'après les autres», comme Faure le lui reprochait. Martine REID emprunte la «petite porte» des Affairements éditoriaux (pp.65-80) de l'écrivain débutant, soit une centaine de lettres qui permettent de comprendre sa singulière démarche auctoriale et de préciser sa situation littéraire. Déjà préoccupé par une censure en alerte, Beyle est soucieux, en «homme bien né», de ne pas publier sous son nom, mais aussi parce que l'identité fictive le préserve d'avoir à «parler comme auteur» (1 ${ }^{\text {er }}$ décembre 1817), même s'il veut montrer qu'il est prêt à s'assurer une place dans le champ littéraire. À cette époque de tâtonnements, le livre résulte d'un travail associant Faure, Crozet et Mareste, collaborateurs et agents littéraires dont la tâche ne s'achèvera pas avec la publication car le livre appelle un autre livre: il faudra reprendre, refaire, amplifier... Ces stratégies font bien de Stendhal un «homme de lettres", surtout si on entend cette expression au sens d'épistolier, comme le propose Brigitte DIAZ (Stendhal «homme de lettres»: sociabilités épistolaires, pp. 81-99) qui voit dans la lettre une propédeutique au métier d'écrivain - ce qui peut passer pour un poncif et même davantage. Peu liant de nature, Stendhal n'a pourtant pas cessé de stimuler ses correspondants. La lettre fut pour lui un exercice de sociabilité mondaine (un «salon virtuel») et de sociabilité littéraire, d'abord pour rester informé de la vie intellectuelle et politique française pendant ses exils - ce pacte épistolaire se retrouvera à peu de choses près dans la première des Lettres sur Paris -, ensuite comme terrain d'essai car elle sert à «causer littérature» (Flaubert) dans un climat de «commerce critique», avec Faure, Crozet, Mareste, Jacquemont, Mérimée, Custine, etc... Même si elle n'est pas un vrai journal de la création, la correspondance de Stendhal contient force indications sur la genèse d'une œuvre comme l'Histoire de la peinture en Italie. Cette connivence entre amis qui se livrent à des conversations épistolaires sans comédie serait-elle une spécificité de la correspondance masculine? La présence de trois thèmes et le contre-exemple des lettres à Sophie Duvaucel le confirment selon Philippe BERTHIER (Entre hommes, pp. 101-113): d'abord la politique et ses dessous - dans une société où elle est une affaire d'homme -, ensuite le sexe et la chronique scandaleuse, enfin l'argent. Mais la 
correspondance est aussi un commerce intellectuel puisque "Stendhal associe étroitement ses meilleurs amis à l'histoire de son esprit», cette association virant d'ailleurs à la collaboration avec Crozet pour l'Histoire de la peinture, avec Mareste pour De l'Amour. Très peu de confidences sentimentales car l'introspection est réservée au journal. Le discret Stendhal n'exprime ses sentiments qu'à de très rares amis, et sobrement: «Je vous aime réellement et il n'y a pas foule» (à Domenico Fiore). Christof WEIAND distingue l'isotopie des Phénomènes d'accélération dans les lettres de Stendhal (pp. 115-127). Stendhal témoignerait de la nécessité de «faire vite, d'aller vite, et plus vite encore», aussi bien quand il écrit à Pauline qu'à la guerre où, évidemment, l'on n'a pas un moment à soi, et plus tard: qu'on pense au consul ou au touriste qu'exalte l'accélération du monde transformé par la technologie de la vapeur, le télégraphe (bien sûr!) et même le daguerréotype. Daniela Gallo (Arts et lettres: le regard de l'épistolier, pp. 129-144) considère cette correspondance comme une source historiographique. On comprend mieux le projet de l'Histoire de la peinture en suivant la lente métamorphose de l'administrateur du musée Napoléon en bon historien de l'art. Ses attitudes et ses réactions face à Rome, ou plutôt l'élaboration subjective de l'image de la Ville à travers ses lettres et celles de ses interlocuteurs permet à Letizia NORCI CAGIANO (L'air de Rome, pp. 145-153) de définir cette impalpable substance à trois époques: la déception d'une mission manquée et premier séjour dont on ne sait pas grand-chose en 1810-1811, les séjours de 1817 (année où il est absorbé par son livre et surtout par Michel-Ange), de 1823-1824 et 1827 (conception des Promenades). Dans la géographie intérieure de Stendhal, Rome a connu une lente cristallisation: d'abord sévèrement jugée («Rome est pourrie»), la Ville fait de sensibles progrès, passant de l'appropriation à la transfiguration. Dans Le consul de France et la politique italienne (1831-1835), François VANOOSTHUYSE (pp. 155-178) réexamine l'organisation du travail décidée par le nouveau consul, dans laquelle on peut voir d'évidentes traces de son passage dans l'administration impériale. Le consul était surveillé, mais il ne manquait pas de protections à Paris et même Rome. On sait moins qu'il avait tendance à croire en la victoire des révolutionnaires alors que ces derniers s'essoufflaient. Diplomate? On peut en douter: si le contenu de ses dépêches est clair, la forme est «ambiguë, ironique, elliptique, insolente». On comprend que Sophie Duvaucel lui ait recommandé d'être «bien sage, bien réservé, bien diplomate en un mot». V. Del Litto jugeait Stendhal un bon fonctionnaire; M. Crouzet a vu dans la correspondance consulaire la marque d'un immense ennui; J.-A. de Sédouy et E. Williamson ont rappelé ses talents d'administrateur. Pour sa part, F. Vanoosthuyse replace la correspondance consulaire dans son contexte politique et dans la trajectoire de Stendhal qui fait de la politique sans tenir compte de la ligne du gouvernement français. Hélène DE JACQUELOT qui prépare une édition critique des Idées italiennes sur quelques tableaux célèbres a complété de documents inédits le corpus de lettres échangées entre Stendhal, Vieusseux, Constantin et quelques autres, d'octobre 1839 à la sortie du livre en août 1840 , soit cent trente lettres et billets dont seulement une vingtaine ont déjà été publiés. Même s'il est resté longtemps dans l'ombre, Stendhal s'est fort impliqué dans la préparation de l'édition. Il a révisé le manuscrit et a eu le dernier mot, tout en tenant compte des «yeux du censeur» que redoutait Vieusseux (Constantin, Stendhal, Vieusseux: une relation triangulaire. Documents inédits, pp. 179-193).

4 Plutôt que de conclure, Elaine WiLliamson (Éditer Stendhal (avec des documents inédits), pp. 195-241) a préféré reconsidérer l'établissement de la correspondance à partir des manuscrits des lettres écrites jusqu'en 1820. Si Stendhal recourt peu fréquemment au 
brouillon, on peut supposer qu'il littérature dans le cas des lettres recopiées dans le journal et inversement, de même pour les documents à quatre mains ou les lettres et rapports rédigés par lui mais signés par une autre personne. E. Williamson pense qu'il n'aurait pas fallu les exclure du corpus de la Correspondance générale et qu'ils devraient figurer dans une édition électronique parce qu'on y retrouve certaines pratiques d'écriture du romancier, par exemple la prise de la voix d'autrui, ou des mécanismes scripturaux repérés dans les manuscrits de Lucien Leuwen, de la Vie de Henry Brulard ou de Lamiel et qui attestent d'une méthode rédactionnelle.

5 Des «années Pauline» aux premières années à Civitavecchia, ce recueil qui multiplie les approches du matériau épistolaire - on regrettera que la politique sous la Restauration fasse figure de parente pauvre - contribue à enrichir la réflexion d'ensemble entreprise par Brigitte Diaz dans Stendhal en sa correspondance ou «l'histoire d'un esprit». 